Argumentos de Mattessich (2003) hacia Macintosh et al. (2000) según artículo: la representación contable y el modelo de capas-cebolla de la realidad: una comparación con las "ordenes de Simulacro" de Baudrillard y su hiperrealidad.

Autor: María Eugenia Quintero Bazán.

Para profundizar el tema te invitamos a leer el número 20 de la Revista Visión Contable:

DOl: http://dx.doi.org/10.24142/rvc.n20a6

El artículo presentaba la postura de dos autores: Mattessich y Macintosh, donde cada expositor expresa su teoría sobre la realidad con respecto a los ingresos y el capital de las organizaciones.

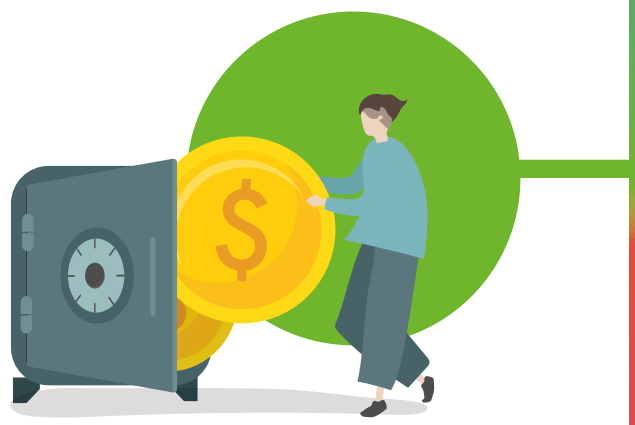

Por otro lado exponen que el termino ingreso, podría ser una representación poco real, ya que este es susceptibles a grandes cambio, puesto que tiene una dependencia o relación con el mercado, intereses y preferencias de las personas o consumidores. Por lo tanto se expone una desactualización en la simbología de la actualidad y referentes de la contabilidad.

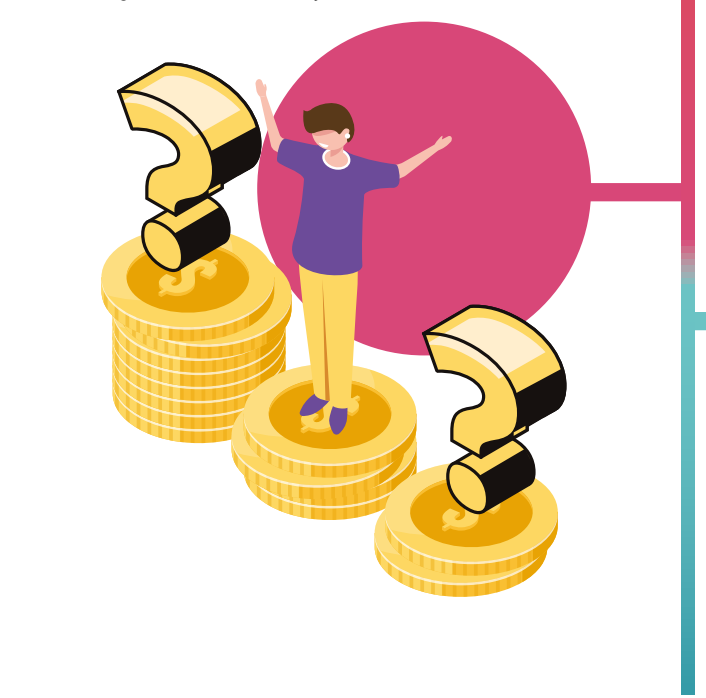

Una de las críticas que plantean es que no reflejan la realidad de las compañías ya que la valoración y medición de estos puede cambiar o variar, dependiendo de las necesidades y políticas que cada empresa tenga, por lo tanto considera que no es prudente utilizar dicha terminología.

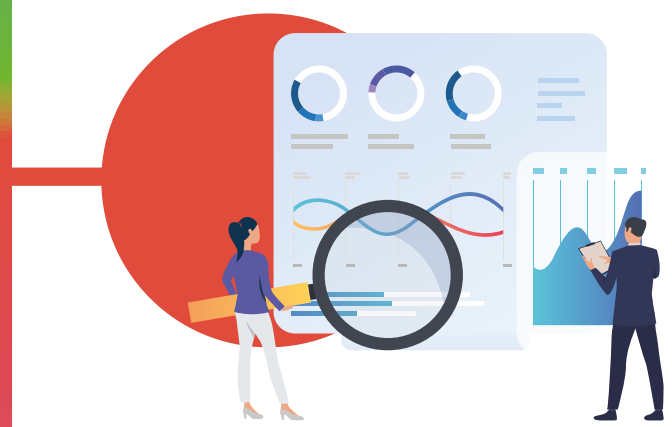

Por ultimo plantean que la contabilidad y, los términos ingreso y capital, podrían ser interpretados o utilizados según los medios y fines que se tenga, pues en ellos se visualizan las alternativas que se presentan al momento de preparar y presentar la información financiera de una entidad respecto al objetivo planteado.

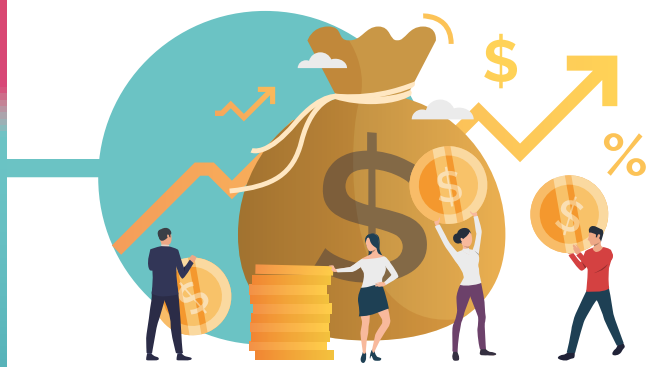

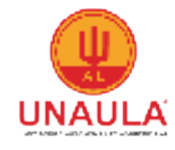
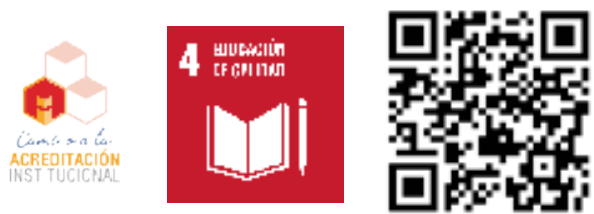


\section{Argumentos de Mattessich hacia Macintosh, Shaerer, Thornyon y Welker sobre la representación contable y la realidad}

María Eugenia Quintero Bazána

Información del artículo
Recibido:02/08/2019
Aceptado: 10/10/2019
Clasificación JEL
M40
Este es un artículo Open Access bajo
la licencia CC BY NC [https://creative-
commons.org/licenses/by-nc/4.0]
Enlace Dol
https://dx.doi.org/DOl: 10.24142/rvc.n20a6
Sugerencia de citación
- Quintero Bazán,M.E.(2019). Argu-
mentos de Mattessich hacia Macin-
tosh, Shaerer, Thornyon y Welker
sobre la representación contable y
la realidad. Revista Visión Contable,
20,156-170. DOl: 10.24142/rvc.
n20a6

Arguments from Mattessich to Macintosh, Shaerer, Thornyon, and Welker on Accounting Representation and Reality

\section{Resumen}

La contabilidad pretende representar de manera fiable la realidad financiera de las entidades. Etimológicamente, la palabra "realidad" proviene del latín y se traduce literalmente como "cosa", en relación directa a la materia física, susceptible de ser observada, estudiada, medida, controlada y transformada. Este artículo presenta, desde una revisión documental, un análisis sobre la realidad en la representación contable, partiendo de la postura de Matessich (2006), en su modelo de Capas-Cebolla de la Realidad, y Macintosh, Shaerer Thornyon y Welker (2000), desde las "órdenes de simulacro" de Baudrillard y su "hiperrealidad". Se concluye que existen dos perspectivas sobre la realidad en la representación contable: la primera recae sobre una representación orientada hacia los objetivos o fines que persigue la información, y la segunda supone una simulación de la realidad por carecer de referentes reales.

\section{Palabras clave}

Contabilidad, realidad, representación.

\begin{abstract}
Accounting aims to reliably represent the financial reality of the entities. Etymologically the word "reality" comes from Latin and literally translates "thing", in direct relation to physical matter, which can be observed, studied, measured, controlled and transformed. This article presents, from a documentary review, an analysis of the reality in accounting representation based on the position of Matessich (2006) in his Layers-Onion model of Reality and Macintosh et al (2000) from the "simulacrum orders "By Baudrillard and his" hyperreality. " It is concluded that there are two perspectives on the reality in the accounting representation, the first one is based on a representation oriented towards the objectives or purposes pursued by the information and, the second one is a simulation of reality because it lacks real references.
\end{abstract}

\section{Keywords}

Accounting, Reality, Representation.

a Licenciada en Contaduría Pública. Licenciada en Administración. Magister Scientiae en Ciencias Contables. Doctora en Ciencias Contables. Docente ordinario en el área de Auditoria en la Universidad Politécnica Territorial del estado Mérida Kléber Ramírez. Coordinadora del Grupo de Investigación en Ciencias Contables de la Universidad Politécnica Territorial del estado Mérida Kléber Ramírez.

Orcid: 0000-0002-0850-6984. maruqbazan@gmail.com 
Argumentos de Mattessich hacia Macintosh, Shaerer, Thornyon y Welker sobre la representación

contable y la realidad

\section{Introducción}

En el trabajo desarrollado por Mattessich (2006), titulado La representación contable y el modelo de capas-cebolla de la realidad: una comparación con las 'órdenes de simulacro' de Baudrillard y su hiperrealidad, se presenta un Macintosh, Shearer, Thornton y Welker (2000) con una postura definida y crítica hacia los actuales conceptos de ingreso y capital, así como de sus referentes reales. Los argumentos presentados por Mattessich (2006) a Macintosh et al. (2000) tienen como punto de partida el modelo de capas-cebolla de la realidad (Mattessich, 1995), sustentado en la orientación hacia un objetivo de la representación contable.

La información presentada como resultado del proceso contable busca estar orientada hacia lo que los usuarios demandan para la toma de decisiones, por tanto, surge la inquietud de preguntarse si la representación contable se orienta hacia un objetivo específico o a representar una realidad que carece de referentes reales. Desde esta perspectiva, se pretende, en este artículo, dilucidar sobre las posturas asumidas por Mattessich (2006) y Macintosh et al. (2000) respecto a este tema tan relevante en materia contable.

La intención del presente artículo recae sobre la reflexión que el profesional contable debe asumir, al momento de presentar información financiera para la toma de decisiones, partiendo de lo que es o no real en un mundo en continuo movimiento. Para ello, el artículo se estructura en un breve recorrido sobre las posturas de Mattessich, y Macintosh, Shearer, Thornton y Welker, así como sus principales discrepancias, para finalmente esquematizar a través de ejemplos contables sus posturas frente a la representación contable.

\section{Argumentos positivos de Macintosh, Shearer, Thornton y Welker}

En época de los token-envelope sumerios, señalan Macintosh et al. (2000), la contabilidad ofrecía una relación clara entre la realidad física y la realidad social en un espacio y tiempo. Esta relación se ha ido fragmentando, y la relación entre el símbolo contable y la realidad que pretende representar hoy día ya no existe, excepto en situaciones contables simples.

Además, Macintosh et al. (2000) consideran que la representación contable, hoy en día, nada tiene que ver con la realidad, lo que remite a simular lo que no se tiene, de allí que su trabajo se apoya en el concepto de 
"hiperrealidad" de Baudrillard y sus "órdenes de simulacro" aplicados a la contabilidad. De la misma manera, los autores abrazan el término "simulación” de Baudrillard desde la óptica de símbolos, imágenes y modelos que circulan a cualquier objeto material real. El eje central de su obra se orienta hacia los símbolos de ingreso y capital, y considera que, en el orden de simulación, la distinción entre el símbolo ingreso y alguna realidad subyacente ha dejado de existir.

Ahora bien, Macintosh et al. (2000) limitan su adecuación hacia la postura baudrillardiana señalando que, si bien los símbolos de la contabilidad, ingreso y capital no reflejan de manera transparente la realidad, la combinación de estos se puede relacionar con la teoría de valoración, sin la necesidad de establecer una definición estricta para estos símbolos.

Por otra parte, el mismo texto (Macintosh et al., 2000) muestra dos alternativas para lograr "exogeneidad y estabilidad en la información contable". En primer lugar, señala la vía relacionada con la escogencia de reguladores a los que llama "miopes", que, siendo conscientes de esta situación, consideran como variables exógenas los datos contables y, a partir de ellos, los toma como medios para la interacción comercial y social, independientemente de sus referentes reales. En segundo lugar, considera a unos reguladores que actúen racionalmente respecto a la arbitrariedad de los estándares definidos.

Para estos autores, los reguladores no pueden intervenir sobre los modelos de valoración de los usuarios al cambiar la descripción de símbolos contables como el ingreso y capital. La posibilidad de elegir unos estándares más beneficiosos que otros dependerá de quién presente la información, pero la escogencia no es porque se refleje una realidad más profunda, sino por ser parte de una realidad existente.

Otro elemento que destacan es lo correspondiente a la "falta de transparencia" en la práctica contable actual y, a través de ejemplos, ilustran que el valor correspondiente a los símbolos contables, como el ingreso, dependen del mercado y del interés o preferencia personal, demostrando así la irrealidad del símbolo ingreso.

De la misma manera, Macintosh et al. (2000) hacen referencia a la "administración de los beneficios", la cual consideran mucho más abusiva $y$, sobre todo, autorreferencial. Esta autorreferencialidad es el punto central de los autores, pues consideran que las teorías de la contabilidad no están preparadas para enfrentar o controlar esta situación, porque se sustentan en 
supuestos desactualizados en relación con los símbolos de la contabilidad y sus referentes reales.

Asimismo, consideran la teoría de la renta global (clean surplus theory o CST, traducido también como teoría de beneficio limpio) y la valoración del ingreso residual como unos de los pilares principales de la contabilidad analítica moderna. Y, en este sentido, argumentan que cambiar la medición de los símbolos contables (ingreso y capital) no tiene ningún efecto en el cálculo de la valoración, siempre que el cambio cumpla la relación del CST.

Finalmente, Macintosh et al. (2000) aseveran que el mundo de los negocios es interpretado por la contabilidad y, a su vez, la contabilidad facilita la interacción, al proporcionar instrumentos arbitrarios -pero exógenospara la contratación y valoración de la actuación.

\section{Réplicas de Mattessich a Macintosh, Shearer, Thornton y Welker}

Mattessich (2006) realiza algunas reflexiones ontológicas partiendo de sorprendentes interrogantes como: “¿qué es real y qué no lo es?”. Para encontrar la respuesta, realiza un recorrido sobre algunos hechos históricos de filósofos como Heráclito, Parménides, Platón y Aristóteles, entre otros, y reconoce en sus teorías la existencia de diferentes capas de la realidad. De allí que fundamente el modelo de capas-cebolla de la realidad (OMR), donde la realidad puede concebirse como una cebolla, en la cual se contemplan diversas capas que representan distintos niveles dentro del contexto de una filosofía de realismo. El propósito del modelo es auspiciar una mejor comprensión de la realidad, así como de la naturaleza de la representación conceptual y lingüística, en relación con nuestras nociones lógicas y percepciones científicas.

El modelo distingue entre realidad absoluta y realidades superiores. En este contexto, prevé la existencia de un núcleo, denominándolo como realidad absoluta, el cual representael centro firme e incuestionado y con una existencia independiente que representa la realidad definitiva. Las realidades superiores a ese centro firme son descritas como: físico-química, biológica, mental y social, conformadas en forma de capas, representando la presencia de diferentes niveles, a manera de jerarquía de realidades.

Mattessich (2006) manifiesta su preocupación por la adecuación que Macintosh et al. (2000) puedan hacer respecto a la postura de Baudrillard, por no tratar estos aspectos contables; no obstante, considera que dichos 
autores pueden interpretar, de una manera más racional y científica, el análisis que Baudrillard realizó respecto a su "hiperrealidad" y "simulación". Para Mattessich, el grupo de Macintosh refleja una división en cuanto a la adopción del criterio de Baudrillard, pues se encuentran, en su documento, comentarios críticos sobre el enfoque filosófico de este. Finalmente, fija posición respecto al enfoque de este otro autor y afirma que él mismo no se orienta a la investigación contable.

Respecto a las interpretaciones que realizan Macintosh et al. (2000) sobre los token-envelope sumerios, Mattessich (2006) considera la necesidad de resaltar algunos comentarios que permitan esclarecer los puntos de error que dichos autores han dado a sus interpretaciones de este tipo de contabilidad sumeria. Estos comentarios corresponden a la utilización errónea de algunos términos referidos a la arqueología de la contabilidad mesopotámica de Mattessich.

De igual manera, dentro de la literatura contable de Macintosh et al. (2000), Mattessich(2006) afirma que estos presentan un error significativo, respecto a la confusión que presentan entre lo ontológico y metodológico al momento de señalar los referentes reales del ingreso o capital. Así, Mattessich señala que aquellos autores solo admiten que el ingreso es real para situaciones simples, y lo niegan para situaciones más complejas, como las opciones sobre acciones. Ahora bien, la crítica que Mattessich señala corresponde a la confusión entre lo ontológico y lo metodológico, pues la estimación o medición del ingreso es un problema de método y, por lo tanto, no deben confundirse las nociones de ingreso y capital con su valor en un momento determinado.

Los nombres que se designan a conceptos como ingreso y capital son cuestiones de preferencia y convenio, esta proposición que hace Mattessich (2006) corresponde al término "arbitrario" de Macintosh et al. (2000), respecto al ingreso y capital. Considera Mattessich que en la disciplina contable existen diversos nombres para definir sus símbolos, tal es el caso de "beneficio" para el ingreso, y "propiedad" en lugar de capital. En este sentido, el autor no considera el uso del término "arbitrario" para los símbolos de ingreso y capital.

De igual manera, considera que este término, para la definición y cálculo de valores del ingreso y capital no es prudente, por cuanto él mismo no representa nada en términos absolutos, sino solo una cuestión de grados: lo que para una persona no consciente del objetivo planteado puede ser arbitrario, para otra conocedora del objetivo no lo es; por tanto, la orienta- 
ción hacia el objetivo y las relaciones medios-fines son de gran importancia (Mattessich, 2006). Cada vez que tomamos alguna decisión estamos conscientes de ello, por tanto, existe un cierto grado de racionalidad, un criterio o un propósito sobre el cual se toma una decisión, y no es una cuestión de arbitrariedad por parte del sujeto que decide.

Respecto a la falta de transparencia en la práctica contable que señalan Macintosh et al. (2000) y que relacionan con la irrealidad del símbolo ingreso, Mattessich (2006) considera que esta es causada no porque el ingreso no sea una verdadera realidad, sino por la negligencia o ausencia de criterios orientados hacia el objetivo perseguido, además de no existir una única manera de medir o estimar el valor del ingreso para todos los objetivos que persigue la información.

Mattessich (2006) fija posición sobre los ejemplos de valoración del ingreso y capital señalados por Macintosh et al. (2000): piensa que los mismos no demuestran la debilidad que los conceptos de ingreso y capital tienen respecto a la representación de los derechos sociales y legales reales, sino que solo muestra cómo el mercado y las fuerzas de poder ejercen influencia sobre la determinación de los valores para reflejar la verdadera realidad que existe en las cifras presentadas.

En cuanto a la autorreferencialidad, que es un punto central de Macintosh et al., estos autores consideran que el símbolo contable precede al referente que intenta representar, y no representa lo verdadero, sino lo que pretende, es su propia simulación considerando referencias circulares respecto a sus propias teorías. Mattessich (2006) considera que no debe confundirse la autorreferencialidad con la circularidad, pues el problema no corresponde ni a la representación conceptual ni a la realidad del símbolo, así como tampoco a la teoría contable, solo atañe a la política en que se establecen los estándares contables.

La satisfacción de Macintosh et al. (2000) respecto a la aceptación por excelencia de la teoría de la renta global (clean surplus theory o CST), para la comprobación de que el ingreso y el capital no tienen referentes en la realidad social, hace pensar a Mattessich (2006) que se confunde de nuevo el tema ontológico con el metodológico y de medición. Las cantidades atribuidas al capital y al ingreso corresponden a aspectos meramente metodológicos, mientras que el derecho sobre el capital y el ingreso pertenece a una realidad socialindiscutible. 


\section{Evaluación de las críticas de Mattessich}

La postura de Macintosh, Shearer, Thornton y Welker (2000), al adoptar los fundamentos filosóficos de Baudrillard respecto al concepto y significado que adquiere la noción de realidad y su aplicabilidad a la contabilidad, es criticada apropiadamente por Mattessich, pues reconoce dicho autor no es un teórico de referencia sobre aspectos contables, además de considerarlo un filósofo meramente literario, metafórico y frecuentemente excéntrico. Baudrillard es considerado por destacados filósofos -como Bunge, Levin y Gane, entre otros- como un filósofo que presenta ensayos cargados de paradojas inauditas, contraproducentes, esotéricas y que no representan ninguna ventaja al sentido común académico.

Respecto a la réplica de Mattessich sobre el uso que el grupo de Macintosh ha dado a sus interpretaciones de la contabilidad mesopotámica y los términos utilizados en su arqueología, los consideró apropiados de acuerdo a la explicación detallada que hace respecto.

La crítica que Mattessich hace a Macintosh, Shearer, Thornton y Welker, respecto a la confusión entre lo ontológico y metodológico, se valida en la situación presentada por este grupo al reconocer los derechos de propiedad como el resultado de las relaciones sociales que determina el valor-signo, independientemente de las consideraciones reales. Mattessich encuentra en esta situación un problema no ontológico, sino metodológico, pues lo que se discute es la valoración, medición o estimación del ingreso.

Otro elemento considerado dentro de las críticas de Mattessich es el término "arbitrario" que el grupo de Macintosh atribuye a los símbolos de la contabilidad. En este contexto, la crítica de Mattessich gira entorno a señalar que no existe tal arbitrariedad, pues lo que se requiere es orientar hacia un objetivo, establecer medios-fines y que exista un cierto grado de racionalidad para tomar decisiones. Considera, además, que lo único arbitrario que existe es el nombre que se le pueda atribuir al símbolo contable (ingreso o capital), lo cual es una cuestión de preferencia y convenio. De igual manera, la selección del método para el cálculo de los valores del ingreso y capital obedece a un propósito o criterio sobre el cual se toman decisiones, y no a la arbitrariedad. Esta situación obedece a una crítica favorable que Mattessich hace sobre la posición que fijan dichos autores respecto al término "arbitrario", y la definición y cálculo de los valores de ingreso y del capital.

Finalmente, la posición asumida por Macintosh et al. (2000) de simular lo que no se tiene cuando se habla de representación contable hoy en día, 
Argumentos de Mattessich hacia Macintosh, Shaerer, Thornyon y Welker sobre la representación contable y la realidad

es criticada acertadamente, pues, para Mattessich (2006), la representación contable está relacionada con la realidad y considera que esta tiene una base empírica a la par de los objetivos señalados en la norma contable.

\section{Análisis de ejemplos contables}

La aplicación del modelo de capas-cebolla de la realidad (OMR) a la contabilidad, presentada por Mattessich (2006), destaca la importancia de reconocer el nivel o subnivel de la realidad social humana en la contabilidad. Como parte de esa realidad social y de las propiedades emergentes se encuentran las obligaciones sociales, los derechos de propiedad y la consciencia, entre otras. En la contabilidad todo gira alrededor de los derechos de propiedad como parte de una realidad social, y es allí donde Mattessich, a través de su ejemplo del manzano, demuestra que, si un derecho de propiedad es real, el ingreso es real; por tanto, el ingreso es real si es consecuencia de un derecho de propiedad o derivado del trabajo o donaciones.

A través de este ejemplo, según explica Mattessich, se desprende una realidad social como es el derecho de propiedad; en este sentido, el ingreso está representado por el derecho que se tiene de la propiedad (árbol), en relación a la cosecha que se ha producido. Las manzanas representan solo la manifestación física del ingreso y, si el derecho sobre el árbol existe, el ingreso será real. Finalmente, este ejemplo representa la relación que debe existir entre el derecho de propiedad y el ingreso, para que este sea considerado como real.

Respecto a la posición asumida por el grupo de Macintosh, en relación con la falta de transparencia en la práctica contable, producto de la inexistencia de una única y adecuada manera para medir o estimar el valor del ingreso para algunos fines, se encuentra un ejemplo referido a la valoración correspondiente a la concesión de opciones sobre acciones a directivos.

En este ejemplo, el grupo de Macintosh ilustra cómo los valores correspondientes al ingreso pueden variar dependiendo del mercado y de las preferencias personales, al momento de seleccionar el método para su valoración, y dependiendo de los fines que se persigan. A través de este ejemplo, se pretende demostrar que los conceptos de ingreso y capital presentan debilidades respecto a su referente real; sin embargo, este propósito no se alcanza, ya que se visualiza una confusión entre lo ontológico y lo metodológico.

El ejemplo describe dos métodos de valoración correspondientes a la concesión de opciones sobre acciones a directivos: el primero considera el 
valor intrínseco como una forma tradicional de valoración, y el segundo reconoce un valor razonable para su contabilización. Así, se trata de demostrar que, dependiendo del método que se escoja, se representa un determinado ingreso, el cual es considerado irreal. Ahora bien, la descripción que señala el grupo de Macintosh en este ejemplo corresponde a la forma como se determina el ingreso; es decir, un procedimiento metodológico que no debe ser confundido para reconocer un ingreso como real o no.

\section{Ejemplos contables}

La representación tiene que ver con la realidad, una realidad que, según Mattessich, puede ser físico-química, biológica, social y mental. La representación contable, vista por este autor, obedece a una realidad fundamentalmente social y mental, la cual está orientada hacia la consecución de un fin.

Ahora bien, la valoración contable es un elemento de gran importancia al momento de considerar la realidad en la representación contable, donde cada proceso de valoración es una realidad social que proviene de una realidad mental de las particularidades de los actores de una sociedad, que influyen de manera significativa en los precios de mercado. Estas particularidades ocasionan ciertas dificultades en la valoración contable, por lo que se hace necesario determinar qué método de valoración es el más adecuado de acuerdo al fin que se persigue y, de esta manera, minimizar la subjetividad que puede estar presente en la representación contable.

Mattessich presenta diversas situaciones en las que se vislumbra cierta dificultad respecto a la valoración. En primer lugar, describe una situación en la que un precio de mercado pagado en una fecha determinada es preciso, señalando la necesidad social de valoración en lapsos posteriores a su transacción. Si se considera la compra de un terreno a un valor de mercado en una fecha determinada, este valor puede verse alterado por situaciones ajenas a la entidad que lo adquirió, como, por ejemplo: invasiones, expropiaciones, inflación, entre otros, situaciones que originan la necesidad social de valoración en períodos posteriores a su transacción o reconocimiento inicial.

En segundo lugar, plantea un escenario en el que se desea determinar el valor de un objeto, así como las distintas alternativas de procedimientos para valorar. Respecto a esta situación, los actuales estándares internacionales señalan, en relación con un edificio, diversas alternativas para deter- 
minar su valor. La Norma Internacional de Contabilidad 16. Propiedades, Planta y Equipo (2008) describe dos métodos de valoración para todos los componentes de propiedades, planta y equipo; por ser el edificio un elemento correspondiente a este rubro, los contadores públicos tienen dos alternativas para su medición, posterior al reconocimiento, estos son: modelo del costo y modelo de revaluación. La selección del modelo obedece al juicio y criterio profesional que se tenga en la entidad, por lo que, tal como señala Mattessich, las opiniones y juicios relacionados con el valor juegan un papel significativo.

Si la política de la entidad para valorar propiedades, planta y equipo se orienta al modelo del costo, la entidad valorará el edificio por su costo, menos la depreciación acumulada y el importe acumulado de las pérdidas por deterioro del valor. Pero si la política de la entidad se orienta al modelo de revaluación, el edificio se valorará a su valor razonable (que pueda ser medido con fiabilidad), en el momento de la revaluación, menos la depreciación acumulada y el importe acumulado de las pérdidas por deterioro de valor que haya sufrido.

En tercer lugar, describe una situación referida a la dificultad que se presenta en establecer el valor de una mercancía concreta y, por tanto, es necesario asignar su valor para un grupo de objetos. Bajo este escenario, puede señalarse como ejemplo una entidad que opera en el transporte turístico terrestre y presta servicio en un número determinado de rutas en distintas temporadas del año (altas y bajas). Cada uno de sus activos puede ser identificado con una ruta turística y se pueden determinar los flujos de efectivo para cada ruta en particular. La rentabilidad de cada una de ellas, en cada temporada, suele variar de manera significativa. Por tanto, en este ejemplo y de acuerdo a la situación planteada, el valor no puede ser determinado para cada una de las unidades de transporte turístico terrestre ni para cada una de las rutas turísticas, de manera que la entidad debe considerar como unidad generadora de efectivo la sumatoria de todos los flujos que generan las distintas unidades turísticas terrestres en las diversas rutas.

Por otra parte, la necesidad que tiene la entidad de valorar estos activos al cierre del ejercicio económico requiere determinar la existencia o no del deterioro de valor que las unidades de transporte turístico terrestre puedan presentar a la fecha de cierre. Para ello, la norma contable actual prevé el método del valor de uso, a través del cual se puede hallar su valor al final del ejercicio (valor recuperable), para luego comparar este con el valor registrado en libros, y determinar cuál es el menor valor entre el resultado 
obtenido aplicado el método y el costo histórico registrado. Esta situación permite visualizar las alternativas de selección que la norma contable actual presenta para el registro fiable de los activos de una entidad.

Estas situaciones expuestas por Mattessich y ejemplificadas en el presente artículo, respecto a las dificultades que se presentan para valorar correctamente los precios de mercado, permiten reconocer que la selección del método de valoración dependerá del propósito específico que se pretenda representar en la contabilidad. La neutralidad de la valoración tiene inmersa la subjetividad, y esta se neutraliza por el objetivo que se persigue. Así, el enfoque que fija Mattessich, en torno a la orientación hacia un objetivo, las relaciones medios-fines y el enfoque condicional normativo, conduce a fijar posición respecto a la importancia que tiene el criterio o propósito al momento de tomar decisiones.

En la actualidad, las normas internacionales de información financiera para pymes, en la sección número cuatro, señala dos alternativas de presentación para el estado de situación financiera: el primero corresponde a una presentación de cada una de las categorías del activo y pasivo como corriente y no corriente, y la segunda alternativa, de acuerdo al orden de liquidez de cada una de las categorías.

La escogencia de una alternativa u otra dependerá del propósito que persiga demostrar la entidad con la información. Si el fin que persigue la entidad es revelar su liquidez a una fecha determinada, como por ejemplo una institución financiera o una entidad que desea invertir en otra entidad, presentará su estado de situación financiera de acuerdo al orden de liquidez, mostrando en el primer rubro del activo la partida más representativa de su liquidez a la fecha de su presentación. Si la situación es contraria, y su propósito es presentar la información de acuerdo al rubro más sólido que tiene la entidad, la norma le brinda la alternativa de presentar la información de acuerdo a la clasificación de partidas como no corrientes; es decir, presentará en el estado de situación financiera, como primera partida, el activo principal que sea considerado como no corriente.

Otro aspecto señalado por Mattessich, respecto a la posición asumida por el grupo de Macintosh, en relación al valor contable a depreciar en el futuro y su incidencia en que todas las ganancias futuras aumentarían por la misma cantidad, se puede ilustrar a través del siguiente ejemplo.

Supongamos que una entidad adquirió un edificio por el que desembolsó la cantidad de cinco millones de bolívares; además, incurrió en mejoras que permitirían poner dicha edificación en condiciones de uso por un millón 
de bolívares. El valor contable a depreciar en el futuro será de seis millones de bolívares, los cuales serán depreciados en un estimado de veinte años. Si al cierre del primer ejercicio económico la entidad muestra una ganancia de setecientos mil bolívares, después de haber disminuido por concepto de gastos de depreciación la cantidad de trescientos mil bolívares, la cual no representó salida de dinero, nos llevaría a analizar que la ganancia real de la entidad fue afectada negativamente por el gasto de depreciación, por lo que la ganancia de la entidad fue de un millón debolívares.

Si el gasto real por depreciación para cada año disminuye el valor contable del edificio en trescientos mil bolívares, al transcurrir los veinte años estimados para su depreciación, las ganancias futuras aumentarían en la misma cantidad en la que se haya depreciado el activo.

\section{Mattessich y Searle ante la existencia de un mundo real}

Para Mattessich y su modelo OMR, la realidad puede concebirse como una cebolla con sus distintas capas, donde cada una de ellas representa una jerarquía de diferentes niveles dentro de un contexto filosófico de realismo. Los términos "existencia" y "real" son significativos para el autor, por lo que se hace necesario distinguir en qué nivel de realidad se consideran términos válidos y en cuáles no. Así, John Searle (1997) señala que, si él no ha demostrado la existencia de un mundo real, su interlocutor confirma la existencia de tal mundo desde el momento en que se comunica con el autor o con cualquier otra persona.

Ambos autores distinguen la existencia de una realidad, Mattessich la reconoce cuando, a través de su modelo OMR, pretende demostrar una mejor comprensión de la noción de realidad, y para ello establece una distinción entre realidad absoluta (realidad definitiva) y realidades superiores. Sobre estas últimas desarrolla su modelo, considerando cuatro tiposde realidades superiores: físico-química, biológica, mental (humana) y social. Estas realidades obedecen a una jerarquía de niveles dentro del contexto de realismo (externo). Por su parte, Searle reconoce una realidad existente, pero no señala un modelo, como Mattessich, que establezca capas como las de una cebolla, solo reconoce una jerarquía de realidades.

En este contexto de realidades, Searle considera que los rasgos fundamentales del mundo están descritos por la física, la química y el resto de las ciencias naturales. Lo más interesante para él es cómo se relacionan las diversas partes, cómo está conformado el mundo entre sí. De esta manera, 
Searle trata de desarrollar una teoría general de la ontología de los hechos sociales y de las instituciones sociales: su enfoque persigue una meta ontológica.

\section{Conclusiones}

Luego de conocer los argumentos de Mattessich (2006) y los de Macintosh et al. (2000), respecto a la realidad de la representación contable, se encuentra que el primero ve la realidad como un conjunto de capas que obedecen a una jerarquía que va desde la realidad absoluta hasta la social. Su análisis se fundamenta en un modelo denominado capas-cebolla de la realidad, a través del cual trata de explicar la realidad de dos conceptos contables como son el ingreso y el capital.

El modelo distingue entre realidad absoluta y realidades superiores. En este contexto, prevé la existencia de un núcleo denominado realidad absoluta, el cual representa el centro firme e incuestionado, y con una existencia independiente que representa la realidad definitiva. Para Mattessich, la representación contable se orienta hacia un objetivo, donde la relación medios-fines debe estar correctamente definida. Considera, además, que debe existir una distinción entre la representación conceptual pragmática y la positiva; sin embargo, si se considera la contabilidad como pragmática, ¿por qué hay que pedirle información positiva a la contabilidad?

Para Macintosh, Shearer, Thornton y Welker, la contabilidad ofrecía una relación clara entre la realidad física y la realidad social, en un espacio y tiempo determinados. Esta relación se ha ido fragmentando y -según los autores- la relación entre el símbolo contable y la realidad que pretende representar hoy día ya no existe, excepto en situaciones contables simples. El grupo de Macintosh se apoya en el concepto de "hiperrealidad" de Baudrillard, así como de sus "órdenes de simulacro", aplicados a la contabilidad, y de allí concluye que la representación contable, actualmente, nada tiene que ver con la realidad, lo que remite a simular lo que no setiene.

Otro aspecto considerado por estos autores corresponde al problema de medición del ingreso, lo que origina valores incorrectos a entes reales en la información presentada. Esta situación es tratada como un problema ontológico y no como uno metodológico, situación que se convierte en una réplica significativa por parte de Mattessich. Al respecto, Searle reconoce una 
realidad existente cuando confirma que su interlocutor se ve comprometido con su existencia al momento de comunicarse con él o cualquier otro ser, y reconoce, así, una jerarquía de realidades.

Como parte final de estas conclusiones, es importante señalar, de acuerdo a la posición fijada por Mattessich respecto a la relación medios-fines, que esta representa un gran reto para la investigación contable. Hoy se habla de una crisis en la representación de la información contable, y existen posiciones radicales como las de Macintosh et al. (2000), que asumen que los símbolos contables no tienen referentes reales y, por tanto, suponen una simulación de la realidad.

Representa un reto para la academia fortalecer la investigación contable en el desarrollo de nuevo conocimiento respecto a la relación medios-fines que fortalezcan la teoría contable actual. Sin embargo, se reconoce el esfuerzo que los reguladores contables han puesto de manifiesto en los estándares internacionales de información financiera, pues en ellos se visualizan las alternativas que se presentan al momento de preparar y presentar la información financiera de una entidad respecto al objetivo planteado. 


\section{Referencias}

Macintosh, N. B., Shaerer, T., Thornyon, D. B. y Welker, M. (2000). Accounting as simulacrum and hyperrealitu: perspectives on income and capital. Accounting Organizations and Society, 25, 13-50.

Mattessich, R. (2006). La representación contable y el modelo de capas-cebolla de la realidad: una comparación con las 'órdenes de simulacro' de Baudrillard y su hiperrealidad. Buenos Aires: CIECE. Recuperado de http://157.92.136.59/download/ docin/docin_ciece_001.pdf

Norma Internacional de Contabilidad 16. Propiedades, Planta y Equipo (2008). Recuperado de https://www. mef.gob.pe/contenidos/conta_publ/ con_nor_co/vigentes/nic/16_NIC. pdf

Searle, J. (1997). La construcción de la realidad social (Trad. A. Doménech). Barcelona: Paidós. 